Original Research Paper

\title{
Big Data Driven Supply Chain Management and Business Administration
}

\author{
${ }^{1}$ Lidong Wang and ${ }^{2}$ Cheryl Ann Alexander \\ ${ }^{I}$ Department of Engineering Technology, Mississippi Valley State University, USA \\ ${ }^{2}$ Technology and Healthcare Solutions, Inc., USA
}

Article history

Received: 30-03-2015

Revised: 08-05-2015

Accepted: 15-06-2015

Corresponding Author:

Lidong Wang

Department of Engineering

Technology, Mississippi Valley

State University, USA

Email: 1wang22@students.tntech.edu

\begin{abstract}
Big Data helps improve visibility throughout the supply chain, provides an integrated view of operational performance and customer interaction and gives businesses real-time insights that help make critical decisions. Big Data also has a potential to yield new management principles. This paper introduces the Big Data concept, its characteristics and some major issues of Big Data in supply chain management and business administration. These issues include supply chain and business data, Big Data benefits and its applications and opportunities. Methods and technology progress about Big Data are presented in this study. General challenges of Big Data and Big Data challenges in supply chain management and business administration are also discussed.
\end{abstract}

Keywords: Big Data, Supply Chain Management, Business Administration, Big Data Analytics, Data Collection, Risk Management, Marketing, Consumer Services, Data Mining, Hadoop

\section{Introduction}

\section{Big Data and Characteristics}

Big data is data that exceeds the processing capacity of conventional database systems. The data is too big, moves too fast, or doesn't fit the strictures of conventional database architectures (Dumbill, 2013). Wal-Mart generates 2.5 petabyte (PB: approximately $10^{15}$ bytes) of customer transaction data every hour. Furthermore, if Wal-Mart operates Radio Frequency Identification (RFID) on the item level, it is expected to generate 7 terabytes (TB: approximately $10^{12}$ bytes) of data every day (Zaslavsky et al., 2012). Devices such as smart phones, webcams, RFID readers and sensor networks add a huge number of autonomous data sources. These devices continuously generate data streams without human intervention, increasing the volume and velocity of data aggregation. The majority of the data is unstructured and contributes to a much larger variety of data types (Jeske et al., 2013).

Big Data characteristics can be described by "6Vs". They are: Volume, Velocity, Variety, Value, Variability and Veracity (Russom, 2011; Eaton et al., 2012; O'Reilly Radar Team, 2012; Zikopoulos and Eaton, 2012; Bellini et al., 2013; Demchenko et al., 2013; Megahed and Jones-Farmer, 2013; Rajpathak and Narsingpurkar, 2013).
Volume: Data is large in the amount such as Petabytes (PB), Zettabytes (ZB: approximately $10^{21}$ bytes) and Yottabyte (YB: approximately $10^{24}$ bytes), etc.

Velocity: Data is generated at a high speed.

Variety: This means increased data type disparity, such as structured data from relational tables, semi-structured data from key-value web clicks and unstructured data from networking data, streamed video and audio.

Value: It means that there is some valuable information-golden data to extract within the collected data, though most of the pieces of data individually may seem valueless.

Variability: It refers to data changes during processing and lifecycle. Increasing variety and variability also increases the attractiveness of data and the potentiality in providing unexpected, hidden and valuable information.

Veracyity: It includes two aspects: Data consistency (or certainty) and data trustworthiness. The following aspects help ensure data veracity:

- Integrity of data and linked data (e.g., for complex hierarchical data, distributed data)

- Data authenticity and (trusted) origin

- Identification of both data and source

- Computer and storage platform trustworthiness 
- Availability and timeliness

- Accountability and reputation

Big data means more information, but it also means more false information. Its focus is on correlations, not causality. It is about what, not why (Bottles et al., 2014). In addition, the data we consider big today may not be considered big tomorrow because of the advances in data processing, storage and other system capabilities (Zaslavsky et al., 2012).

\section{Supply Chain and Business Data}

Supply Chain Management (SCM) data science uses quantitative and qualitative methods from various disciplines in combination with SCM theory to predict outcomes and solve relevant SCM problems. SCM predictive analytics uses both quantitative and qualitative methods to improve supply chain design by predicting past and future levels of the integration of business processes among companies, as well as the associated costs and service levels (Waller and Fawcett, 2013a).

Achieving a competitive level of global supply chain excellence cannot be achieved without data-driven, endto-end operations. Manufacturers and retailers collect data all along their supply chains. This includes data from Point of Sale (POS), Global Positioning System (GPS) and RFID data, to data emitted by equipment sensors, or social media feeds. Walmart is an early adopter of data-driven supply chains. It optimizes all its supply chain decisions-from customer fulfillment to inventory tracking and automatic purchase (Sanders, 2014). Big Data has the potential to revolutionize supply chain dynamics and business management. In supply chain and business management, the great value of big data often comes from integrated data sources and actionable information. Some examples of big data emerging supply chains and business are shown in Table 1.

Table 1. The Big Data emerging in supply chain and business (Cecere, 2012)

\begin{tabular}{ll}
\hline Structured Data & Transactional data \\
& Time phased data \\
Snstructured Data & Cocial \\
& Channel \\
& Customer service \\
Sensor Data & Warranty data \\
& Temperature \\
& RFID \\
QR codes \\
New Data Types & GPS \\
& Mapping and GPS \\
& Video \\
& Voice \\
& Digital images \\
\hline
\end{tabular}

*QR code: Quick Response code, the trademark for a type of matrix barcode

\section{Benefits of Big Data in Supply Chain Management and Business Administration}

Big Data has the potential to improve effectiveness and efficiency, produce higher-quality outputs and increase the value-added content of products and services. Big data analytics has given rise to intelligent supply chains (Sanders, 2014). Big Data offers the following benefits in supply chain management and business administration (Brown et al., 2011; Zaslavsky et al., 2012; Jeske et al., 2013; Trimarco and Stark, 2013).

More accurate information, improved visibility and improved product quality:

- Provide more accurate operational information and enable timely correction or supplier change

- Improve transparency of information and give greater visibility throughout the supply chain

- Improve product and service traceability

- Better identify potential problem suppliers as well as identify problems for suppliers

- Uncover defects in products/services in the supply chain, give early warning and avoid recalls

- Minimize inventory and supply chain risk using big data analytics

Higher operational efficiencies:

- Substantially expedite decision-making based on sophisticated Big Data analytics

- Leverage big data to track and manage delivery in real time by analyzing weather, traffic and location data feeds to determine the exact time of delivery

- Expedite order picking and order fulfillment by analyzing data from different sources like historical orders, product inventory, warehouse layout and historical picking times

- Enable real-time vendor management by reviewing vendor performance including vendor profitability, ontime service and customer feedback and complaints

- Offer a real-time view of the product demand and product sales; and therefore improve and expedite product sourcing process

Personalized service and improved service quality:

- Increase customer engagement

- Analyze customer interactions across all channelssocial, mobile and web-to determine how the customer is using the products they bought or will buy

New business models and better prediction:

- Extract valuable information from real-time multiple source data, build a technical backbone for the 
development and deployment of new data-driven business models

- Spawn new categories of companies that embrace information-driven business models. Many of these businesses play intermediary roles in value chains

- Improve the development of the next generation of products and services

- Generate more revenue and predict future outcomes with greater confidence in low cost

\section{Applications and Opportunities of Big Data in Supply Chain Management and Business Administration}

Big Data can strengthen supply chain and business management in the following areas (Software AG, 2013):

Procurement: Organizations can make better procurement decisions according to more factors.

Supply chain collaboration: All partners understand how every other partner is responding to an event and the responses are fulfilled in concert with each other.

End-to-end supply chain execution: Big data can make organizations seamlessly combine and correlate multiple data sources to ensure end-to-end supply chain execution is finished effectively and efficiently.

Supply chain planning: Big Data uses massive amount of data, taking into account performance variables and leveraging more complicated "what if" scenarios. Manufacturers can improve their demand forecasting and supply planning using their own data. In addition, more value can be unlocked when companies are able to integrate data from other companies and other sources including data from retailers, such as promotion data (e.g., items, prices, sales) and inventory data (e.g., stock levels per warehouse, sales per store) (Software AG, 2013; Chui et al., 2010).

Inventory control: WalMart's inventory management system pioneered the age of big data by enabling suppliers to see the exact number of their products on every shelf of every store at each precise moment in time (Tene and Polonetsky, 2013). In large complex and global automotive supply chains, Big Data analytics will contribute significantly in reducing intransit inventory, safety stocks and stock-outs by better analysis of transit times, bottlenecks at ports and shipment routings (Bhattacharya et al., 2014).

Marketing and sales/after-sales support: There are many opportunities to leverage Big Data in the marketing, sales and after-sales service activities. The opportunities can be the segmentation of customers and applying analytics in order to improve the effectiveness of sales forces (Chui et al., 2010).

Tracking locations: Location-based data can be obtained from four primary sources: In door tracking devices, such as RFID tags on shopping carts; in-person credit or debit card payment data; GPS chips in mobile devices; and cell-tower triangulation data on mobile devices. Location-based services can predict future traffic conditions, based on matching current to historical data, combining it with weather forecasts and information from past traffic patterns, etc. (Hilbert, 2013).

Personalized service: Leveraging big data with predictive analytics improves predictions of individual consumer behavior (Waller and Fawcett, 2013b). In retail business, consumer behavior and preferences can be understood by analyzing the big data which includes customer movement in the store or online webs site, transactions, product searches, etc. (Bucholtz, 2012). Some retailers use Big Data analytics to perform "sentiment analysis", which mines huge streams of data generated by consumers using various types of social media, gauges responses to new marketing campaigns in real time and adjusts strategies accordingly (Brown et al., 2011).

Business Intelligence and Analytics (BI\&A) and the related field of Big Data analytics have become increasingly important in the business communities. Table 2 (Chen et al., 2012) summarizes some BI and A features and capabilities in e-commerce and market intelligence, including applications, data characteristics, analytics and potential impacts. Big Data analytics can also be used to identify deception and deceptive practice to increase e-commerce and supply chain security (Zage et al., 2013).

In a survey, participating companies did not benefit equally from their use of Big Data analytics. Some companies in the survey lived up to its promise helping them improve customer service and demand fulfillment, experience faster and more effective reaction time to supply chain issues, increase supply chain efficiency and drive greater integration across the supply chain. It is shown in Table 3 (Accenture, 2014).

Big Data analytics holds significant promise for improving supply chain management. Analyzing vast amounts of varied data in real time helps companies understand their customer better, reduces costs and helps better manage supply chain risk (Sanders and Ganeshan, 2015). Big Data analytics facilitates enhanced decision making, increased visibility and overall greater value. The applications and opportunities of Supply Chain Management (SCM) predictive analytics are especially seen in the form of more informed decision-making capabilities, ability to improve supply chain efficiencies and enhanced demand planning capabilities (Schoenherr and Speier-Pero, 2015).

Table 4 (Jeske et al., 2013) shows three value dimensions for Big Data analytics. Information transparency and visibility helps achieve high operational efficiency. In customer experience, social influence analysis helps improve customer retention, avoiding "out of stock" conditions for customer satisfaction. In new business models, crowd analytics delivers retail and advertising insights, creating new insurance products from geo-localized data. 
Table 2. BI and A in e-commerce and market intelligence: from Big Data to big impact

\section{Applications}

Data

Analytics

Impacts
Recommender systems

Social media monitoring and analysis

Crowd-sourcing systems

Social and virtual games

Search and userlogs

Customer transaction records

Customer-generated content

Characteristics:

Structured web-based, user-generated

content, rich network information, unstructured informal customer opinions

Association rulemining

Database segmentation and clustering

Anomaly detection

Graph mining

Social network analysis

Text and web analytics

Sentiment and affect analysis

Long-tail marketing, targeted and personalized recommendation, increased saleand customer satisfaction

Table 3. Results companies have achieved using Big Data analytics

\begin{tabular}{ll}
\hline Aspects of achievement & Percentage of companies \\
\hline Improvement in customer service and demand fulfillment of $10 \%$ or greater & 46 \\
Faster and more effective reaction time to supply chain issues & 41 \\
Increase in supply chain efficiency of 10\% or greater & 36 \\
Greater integration across the supply chain & 36 \\
Optimization of inventory and asset productivity & 33 \\
More effective S\&OP process and decision making & 32 \\
Improved cost to serve & 28 \\
Better customer and supplier relationships & 28 \\
Improvement in customer service and demand fulfillment of less than $10 \%$ & 27 \\
Increase in supply chain efficiency of less than $10 \%$ & 26 \\
Improvement in demand driven operations & 20 \\
Shortened order-to-delivery cycle times & 14
\end{tabular}

S\&OP: Sales and Operations

Table 4. Value dimensions for Big Data use cases

\begin{tabular}{ll}
\hline Operational efficiency & Use data to: \\
& Increase level of transparency \\
& Optimize resource consumption \\
Customer experience & Improve process quality and performance \\
& Exploit data to: \\
& Increase customer loyalty and retention \\
& Perform precise customer segmentation and targeting \\
New business models & Optimize customer interaction and service \\
& Capitalize on data by: \\
& Expanding revenue streams from existing products \\
& Creating new revenue streams from entirely new (data) products \\
\hline
\end{tabular}

\section{Methods and Technology Progress around Big Data}

For the development of the intelligent supply chain management system, the big data processing is the found action. In order to improve data processing capability, the Hadoop framework is used to achieve the distribution storage and analysis work of the collected big data. Big data technology improves data processing capability and improves the data value (Yan et al., 2014).

Big Data predictive analytics uses a variety of techniques such as statistics, modeling and data mining to analyze current and historical facts and make predictions for the future. Big Data can fulfill correlation analysis. Correlation analysis points us whether or how two things are connected (Sanders, 2014). 
Management decisions based on data analytic methods are related to data quality. The data quality problem in the context of Supply Chain Management (SCM) was studied; methods for monitoring and controlling data quality were proposed (Hazen et al., 2014). An approach was proposed to provide an analytic infrastructure for companies to incorporate their own competence sets with other companies. The analytic infrastructure was developed and tested based on the deduction graph technique (Tan et al., 2015). Measuring the benefits of Enterprise Resource Planning (ERP) on supply management maturity model was studied based on a "big data" method. This study provides an extensive analysis of how the deployment of ERP systems and the selection of ERP vendors can benefit a company's supply chain performance (Huang and Handfield, 2015).

Big Data has been creating an architectural paradigm shift in data movement. In-memory data analysis with distributed, Massively Parallel Processing (MPP) architectures is gaining momentum. Robust database management and data streaming management capabilities are critical for analyzing streaming data in motion due to the rise of large and continuous streams of data sources (Wixom et al., 2014).

IBM's recent report shows what Big Data tools and techniques are being leveraged as of mid-2013. It highlights these tools and techniques in order of frequency of use from highest to lowest in Table 5 (Schlegel, 2015). This table shows that $91 \%$ of companies use core analytics capabilities such as query and reporting, $77 \%$ use data mining to analyze Big Data and $71 \%$ use data visualization, etc.

Five technologies will make supply chain management more efficient (Zaslavsky et al., 2012). They are: Mobility, Internet of Things (IoT), big data, predictive analysis and cloud computing. These five areas are strongly interconnected. Internet of Things and mobility provide the sensors that can sense in real-time even while moving. These sensors produce big data. Predictive analysis techniques analyze the collected data to extract knowledge. An elastic infrastructure, such as the cloud, needs to be used to perform such techniques over big data. In other terms, the cloud binds to the Internet of Things (Reed et al., 2012). Advances in computing architecture (such as cluster computing and cloud computing) have enabled the storage, retrieval, analysis, sharing and distribution of data easier and cheaper (Sanders and Ganeshan, 2015).

Cloud services have an important role to play in Big Data. The core principles defining what constitutes Big Data in the cloud are presented in Table 6 (Kobielus, 2014).

Table 5. Big data analytics capabilities/tools

\begin{tabular}{ll}
\hline Analytics tools & Percentage of companies \\
\hline Query and reporting & 91 \\
Data mining & 77 \\
Data visualization & 71 \\
Predictive modeling & 67 \\
Optimization & 65 \\
Simulation & 56 \\
Natural language text & 52 \\
Geo-spatial analytics & 43 \\
Streaming analytics & 35 \\
Video analytics & 26 \\
Voice analytics & 25 \\
\hline
\end{tabular}

Table 6. Core architectural principles of cloud-based Big Data deployments

\begin{tabular}{ll}
\hline On-demand self-service & $\begin{array}{l}\text { Enables a Big Data cloud service customer to self-provision cloud } \\
\text { services--both physical and virtual--automatically and with minimal } \\
\text { interaction involving the cloud service provider. }\end{array}$ \\
Enables Big Data cloud resources to be made available over the network and \\
accessible through standard mechanisms by diverse client platforms. \\
Multi-tenancy & $\begin{array}{l}\text { Enables Big Data cloud resources to be allocated so that multiple tenants and } \\
\text { their computations and data can be guaranteed isolation from one another. } \\
\text { Aggregates Big Data cloud resources in a location-independent fashion to serve } \\
\text { mesource pooling }\end{array}$ \\
multiple multi-tenant customers, enabling resources to be dynamically assigned \\
and reassigned on demand and to be accessed through a simple abstraction. \\
Enables Big Data cloud resources to be rapidly, elastically, \\
and automatically scaled out up, out and down on demand. \\
Enables Big Data cloud resources to be monitored, controlled, \\
reported and billed transparently.
\end{tabular}


Besides general cloud infrastructure services (storage, compute, infrastructure/VM management), the following services are required to support Big Data (Turk, 2012):

- Cluster services

- Hadoop related services and tools

- Specialist data analytics tools (logs, events, data mining, etc.)

- Databases/Servers SQL, No SQL

- MPP (Massively Parallel Processing) databases

- Registries, indexing/search, semantics, namespaces

- Security infrastructure (access control, policy enforcement, confidentiality, trust, availability, privacy)

\section{Challenges of Big Data}

\section{General Challenges of Big Data}

The challenges in big data can be broadly divided in to two categories: Engineering and semantic. Engineering challenge is to perform data management activities such as query and storage efficiently. Semantic challenge is to extract the meaning of the information from massive volumes of unstructured dirty data (Bizer et al., 2012).

Some of the inherited challenges in big data are capture, storage, search, analysis and virtualization due to its characteristics (Zaslavsky et al., 2012). Bringing big data from different sources together has been a challenge. Large parts of valuable data lurk in "data silos" of different departments, regional offices and specialized agencies. Fragmentation impedes the massive and timely exploitation of data. Interoperability and integration of isolated data silos and data interoperability standards are becoming pressing issues for Big Data (Hilbert, 2013). Therefore, the following challenges have become important issues (Demchenko et al., 2012):

- Exponential growth of data volume produced by different instruments and/or collected from sensors

- Need to consolidate e-Infrastructures as persistent research platforms to ensure continuity and crossdisciplinary collaboration

In addition, the enhancement of big data with interfaces for user interaction increases the number of access points and correspondingly elevates the risk of data leakage, security breach and privacy (Tene and Polonetsky, 2013). The specific information of some challenges about privacy and information security is as follows (Sethuraman, 2012):
- Different sets of data that were not previously considered as having privacy concerns are combined in ways that threaten privacy

- Hacking or other forms of unauthorized access threatens security

- Data are increasingly collected autonomously, independent of human activity. There are ethical concerns when there is limited or no human involvement

- The combinations of data for which there are currently no capabilities to analyze could become subject to privacy breaches in the future

\section{Big Data Challenges in Supply Chain Management and Business Administration}

RFID allows a sensor to read a unique product identification code associated with a tag. RFID is widely used in inventory management and logistics. However, RFID data are inherently noisy and redundant; RFID data are temporal, streaming and in high volume ( $\mathrm{Hu}$ et al., 2014). Dirty data is a big problem. Some challenges of Big Data in supply chain and business management are provided as follows (Zaslavsky et al., 2012; Issa, 2013; Doherty and Misrahi, 2013):

- It is not easy to analyze multi-channel customer sentiment and experience

- In a global and extended supply chain, ownership of supply chain data is dispersed among many organizations. With no common system or data standards among the organizations, the data requires significant manipulation to be useful

- Fragmentation exists because organizations rarely have complete and integrated supply chain system and data architecture

- Many supply chain stakeholders do not really understand what value they could get from using Big Data

- Much of the potential value comes from sharing data. However, companies that own the data are hesitant to share it. Companies are concerned about that data sharing may result in a loss of competitive advantage

- Shared data requires that there be some neutral data repository that aggregates, organizes and makes the data available in some useful form. However, no such repository currently exists

\section{Conclusion and Future Research}

There are hidden high-valued data mixed with raw and noise data in Big Data. Data value is closely related to the data volume and variety. Big Data technologies can unlock significant value by making information transparent and usable based on Big Data analytics and 
benefit enterprise or organizations. Big Data help improve supply chain management and business administrationin many aspects. These aspects include efficiency, defect identification in products and services, procurement, supply chain collaboration, end-to-end supply chain execution, supply chain planning, inventory control and risk management, marketing and sales/aftersales support, geo-location/mapping, real-time personalized service, optimized merchandising and ecommerce and market intelligence, etc.

Big Data has challenges in supply chain management and business administration. These challenges include data capture, date integration, data visualization, data integration, data sharing, data privacy and information security, highly fragmented data ecosystem and unstructured, unprocessed and seemingly unmanageable data, etc.

All the aspects of these challenges can be future research. The authors of the paper will focus on Big Data in opinion mining, reputation and social influence, personalization mobile advertising and marketing and multilingual analysis for global business.

\section{Acknowledgement}

This research was supported in part by Technology and Healthcare Solutions, Inc. in Mississippi, USA. No conflict of interest to disclose.

\section{Author's Contributions}

Lidong Wang and Cheryl Ann Alexander worked on the paper as group efforts at all stages.

\section{Ethics}

This article is original and contains unpublished material. The corresponding author confirms that both the authors have read and approved the manuscript and no ethical issues involved.

\section{References}

Accenture, 2014. Big Data analytics in supply chain: hype or here to stay? Technical Report, Accenture Company.

Bellini, P., M.D. Claudio, P. Nesi and N. Rauch, 2013. Tassonomy and Review of Big Data Solutions Navigation. In: Big Data Computing, Akerkar, R. (Ed.), CRC Press, pp: 57-101.

Bhattacharya, S., D. Mukhopadhyay and S. Giri, 2014. Supply chain management in Indian automotive industry: Complexities, challenges and way ahead. Int. J. Managing Value Supply Chains, 5: 49-62. DOI: $10.5121 /$ ijmvsc.2014.5206
Bizer, C., P. Boncz, M.L. Brodie and O. Erling, 2012. The meaningful use of Big Data: Four perspectives -- four challenges. ACM SIGMOD Rec., 40: 56-60. DOI: $10.1145 / 2094114.2094129$

Bottles, K., E. Begoli and B. Worley, 2014. Understanding the Pros and cons of Big Data analytics. Physician Executive, 40: 6-12.

Brown, B., M. Chui and J. Manyika, 2011. Are you ready for theera of 'Big Data'? McKinsey Quarterly, McKinsey Global Institute, pp: 1-12.

Bucholtz, C.J., 2012. Big Data and the internet of things.

Cecere, L., 2012. Big Data: Go Big or Go Home? Technical Report, Supply Chain Insights LLC, pp: $1-19$.

Chen, H.C., R.H.L. Chiang and V.C. Storey, 2012. Business Intelligence and Analytics: from Big Data to Big Impact. MIS Q., 36: 165-1188.

Chui, M., M. Löffler, R. Roberts, 2010. The internet of things. McKinsey Q., 2010: 1-3.

Demchenko, Y., Z. Zhao, P. Grosso, A. Wibisono and C. de Laat et al., 2012. Addressing big data challenges for scientific data infrastructure. Proceedings of the 4th IEEE Conference on Cloud Computing Technologies and Science (CloudCom2012), Dec. 3-6, IEEE Xplore press, Taipei, pp: 614-617. DOI: $10.1109 /$ CloudCom.2012.6427494

Demchenko, Y., P. Grosso, C. de laat and P. Membrey, 2013. Addressing big data issues in scientific data infrastructure. Proceedings of the International Conference on Collaboration Technologies and Systems, May, 20-24, IEEE Xplore press, San Diego, pp: 48-55. DOI: 10.1109/CTS.2013.6567203

Doherty, S. and T. Misrahi, 2013. Outlook on the logistics and supply chain industry. World Economic Forum, pp: 1-36.

Dumbill, E., 2013. Making sense of Big Data. Big Data, BD1-BD2.

Eaton, C., D. Deroos, T. Deutsch, G. Lapis and P. Zikopoulos, 2012. Understanding Big Data. McGraw-Hill Companies.

Hazen, B.T., C.A. Boone, J.D. Ezell and L.A. JonesFarmer, 2014. Data quality for data science, predictive analytics and Big Data in supply chain management: An introduction to the problem and suggestions for research and applications. Int. J. Production Econ., 154: 72-80. DOI: $10.1016 /$ j.ijpe.2014.04.018

Hilbert, M., 2013. Big Data for development: From information- to knowledge societies. Technical Report, University of Southern California, Davis, USA.

Hu, H., Y. Wen, T.S. Chua and X. Li, 2014. Toward Scalable Systems for Big Data Analytics: A Technology Tutorial. Access IEEE, 2: 652-687. DOI: 10.1109/ACCESS.2014.2332453 
Huang, Y.Y. and R.B. Handfield, 2015. Measuring the benefits of ERP on supply management maturity model: A "Big Data" method. Int. J. Operations Production Manage., 35: 2-25. DOI: 10.1108/IJOPM-07-2013-0341

Jeske, M., M. Grüner and F. Weiß, 2013. Big Data in logistics. Technical Report, DHL Customer Solutions and Innovation, Germany.

Kobielus, J., 2014. Deploying Big Data analytics applications to the cloud: Roadmap for success. Cloud Standards Customer Council, pp: 1-21.

Schlegel, G.L., 2015. Utilizing big data and predictive analytics to manage supply chain risk. J. Bus. Forecasting.

Issa, N., 2013. Supply chain: Improving performance in pricing, planning and sourcing. Technical Report, Opera Solutions Company.

Megahed, F.M. and L.A. Jones-Farmer, 2013. A Statistical Process Monitoring Perspective on "Big Data", Frontiers in Statistical Quality Control, 11th Edn., Springer, New York, pp: 1-21.

O’Reilly Radar Team, 2012. Planning for Big Data, O'Reilly.

Rajpathak, T. and A. Narsingpurkar, 2013. Managing knowledge from Big Data analytics in product development. White Paper, Tata Consultancy Services, Software AG.

Reed, D.A., D.B. Gannon and J.R. Larus, 2012. Imagining the future: Thoughts on computing. Computer, 45: 25-30. DOI: 10.1109/MC.2011.327

Russom, P., 2011. Big data analytics. TDWI Best Practices Report, Fourth Quarter.

Sanders, N.R. and R. Ganeshan, 2015. Call for Papers: Special Issue of production and operations management on Big Data in supply chain management. Production Operations Management, 24: 354-355.

DOI: $10.1111 /$ poms. 12340

Sanders, N.R., 2014. Big Data Driven Supply Chain Management: A Framework for Implementing Analytics and Turning Information into Intelligence, 1st Edn., Pearson Education, Upper Saddle River, ISBN-10: 0133801284, pp: 262.

Schoenherr, T. and C. Speier-Pero, 2015. Data Science, Predictive Analytics and Big Data in Supply Chain Management: Current State and Future Potential. J. Bus. Logistics, 36: 120-132. DOI: $10.1111 / \mathrm{jbl} .12082$

Sethuraman, M.S., 2012. Big Data's impact on the Data supply Chain. Cognizant 20-20 Insights.

Software AG, 2013. The Value of Big Data in the supply chain: From planning and collaboration to execution, information is power. White Paper, Software AG Company.
Tan, K.H.,Y.Z. Zhan, G. Ji, F. Ye and C.T. Chang, 2015. Harvesting Big Data to Enhance Supply Chain Innovation Capabilities: An Analytic Infrastructure Based on Deduction Graph. Int. J. Production Economics, 166: 223-233. DOI: $10.1016 /$ j.ijpe.2014.12.034

Tene, O. and J. Polonetsky, 2013. Big Data for all: Privacy and user control in the age of analytics. Northwestern J. Technol. Intellectual Property, 11: 239-272.

Trimarco, C.T. and A. Stark, 2013. Supply chain trends impacting the consumer products industry. Technical Report, Capgemini Consulting and Kane Is Able, pp: 1-6.

Turk, M., 2012. A chart of the Big Data ecosystem. Take 2.

Waller, M.A. and S.E. Fawcett, 2013a. data science, predictive analytics and big data: A revolution that will transform supply chain design and management. J. Bus. Logistics, 34: 77-84. DOI: $10.1111 / \mathrm{jbl} .12010$

Waller, M.A. and S.E. Fawcett, 2013b. Click here for a data scientist: Big Data, predictive analytics and theory development in the era of a maker movement supply chain. J. Bus. Logistics, 34: 249-252. DOI: $10.1111 / \mathrm{jbl} .12024$

Wixom, B., L. Iyer, T. Ariyachandra, D. Douglas and M. Goul et al., 2014. The current state of business intelligence in academia: The arrival of Big Data. Communi. Association Inform. Syst., 34: 1-13.

Yan, J., S. Xin, Q. Liu, W. Xu and L. Yang et al., 2014. Intelligent supply chain integration and management based on cloud of things. Int. J. Distributed Sensor Netw., 2014: 1-15. DOI: 10.1155/2014/624839

Zage, D., K. Glass and R. Colbaugh, 2013. Improving Supply Chain Security Using Big Data. Proceedings of the 2013 IEEE International Conference on Intelligence and Security Informatics (ISI), Jun, 4-7, Seattle, IEEE Xplore press, pp: 254-259. DOI: 10.1109/ISI.2013.6578830

Zaslavsky, A., C. Perera and D. Georgakopoulos, 2012. Sensing as a service and big data. Proceedings of the International Conference on Advances in Cloud Computing (ACC), Bangalore, India, pp: 1-8.

Zikopoulos, P., C. Eaton and D. deRoos, 2012. Understanding bigdata: Analytics for enterprise class hadoop and streaming data. McGraw-Hill, New York. 